\title{
Dark Matter in the Milky Way
}

\author{
Chris Flynn \\ Tuorla Observatory, Turku, Finland
}

\begin{abstract}
.
A major uncertainty in studying small groups of galaxies is the total masses of the individual galaxies themselves. I discuss here recent measurements of the mass of the Milky Way disk and new constraints on possible constituents of the putative dark halo.
\end{abstract}

\section{Introduction}

An outstanding problem in the dynamics of galaxies in small groups or otherwise is the total gravitating mass of the galaxies themselves. Rotation curves of spiral galaxies, velocity differences between satellites of galaxies and between members of small groups, and X-ray studies all indicate that the amount of matter present in galaxies appears to exceed the visible matter be factors of a few to an order of magnitude, and has come to be termed the dark matter problem.

Presently, the most accurately measured galaxy mass is that of our own Milky Way. In this paper I summarise recent work on the mass of the Galaxy's disk. These studies indicate that the disk mass locally is now well measured, and that there is no dynamically significant missing matter in the disk. The form and mass of the putative "dark halo" of the Galaxy is currently unresolved and a very active topic, and I summarise recent work in this area. The total gravitating mass of galaxies in small groups or otherwise remains poorly known.

\section{Milky Way Disk Mass}

\subsection{Historical context}

Determining the gravitating mass of the Milky Way near the Sun dates from the wrok of Oort $(1932,1960)$. A range of studies up to the mid 1970's indicated that a significant discrepency between the amount of gravitating matter (as measured by the kinematics and scale height of disk stars through the Poisson-Boltzmann equation; see e.g. Binney and Merrifield 1999) and the amount of visible matter (i.e. in stars, gas and dust). The visible matter appeared to account for only a half to two thirds of the total gravitating matter. We refer to this as the "disk dark matter problem". The history of dark matter has been recently reviewed by van den Bergh (1999) and Einasto and Einasto (2000).

A resurgence of interest in the disk dark matter problem developed in the late 1970's because of the discovery of a much more significant dark matter problem, that of dark matter halos around galaxies. Rotation curves indicate 
that disk galaxies may be heavier than their visible components by as much as a factor of ten, depending partly on the disk mass. As a result there were two dark matter problems on galactic scales, that of the disk (a factor of two or less "missing") and that of the "dark halo". Furthermore, while baryonic or non-baryonic candidates possibly constitute the dark halo, the dark disk component, which would be flattened like the visible disk, must be assembled from dissapative material and hence baryonic only. Two dark matter problems put new emphasis during the 1980's on determining the disk mass with much better accuracy.

By the end of the early 1990's a new generation of surveys were completed, designed specifically for the task of measuring the local gravitating mass of the disk, but they has come to different conclusions; on the one hand, Bienayme et al. (1987) and Kuijken and Gilmore (1989) claimed that the gravitating and visible mass of the disk were the same, at $46 \pm 9 \mathrm{M}_{\odot} \mathrm{pc}^{-2}$ at the Sun in gravitating matter, versus $48 \pm 8 M_{\odot} \mathrm{pc}^{-2}$ in visible matter; while Bahcall, Flynn and Gould (1992) derived a much higher mass estimate of $84 M_{\odot} \mathrm{pc}^{-2}$, albeit with a higher error of $26 M_{\odot} \mathrm{pc}^{-2}$. The evidence now favoured no disk dark matter although the dust had not yet settled on the issue.

\subsection{Hipparcos}

The European Space Agency's Hipparcos satellite was launched in 1989, and measured parallaxes and proper motions for approx 120,000 stars. Complete sky coverage was carried out to approx $V=8$ (but depending on Galactic latitude) for circa 50,000 stars. This complete survey of parallaxes permitted a precise construction of the number density of stars as a function of height $Z$ above the Galactic plane, in the range $-100<Z<100 \mathrm{pc}$. Over this range, the younger stellar populations, $A$ and $F$ stars, show a significant decrease in space density, while the older stellar populations (most G, K and M dwarfs and most giants) show a minor drop in space density.

The 1-dimensional Poisson-Boltzmann equation in the vertical direction $Z$, expresses a simple relation between the kinetic energy of a stellar population (i.e. velocity dispersion, $\sigma_{Z}$ ), the number density falloff with height, $\nu(Z)$ and the total gravitational potential of the system in which the stars reside, $\Phi(Z)$. The density fall off $\nu(Z)$ was by far the most poorly determined part of all preHipparcos analyses of the disk mass. Hipparcos has measured $\nu(Z)$ for the young disk population for the first time directly from the individual stellar parallaxes, and so provides a huge improvement in the disk mass determination.

Two groups have recently determined the disk mass using the Hipparcos data, both arriving at the conclusion that the total gravitational mass is, within measurement error, the same as the visible mass, and hence that there is no significant "disk dark matter problem".

Both groups analysed $\mathrm{A}$ and $\mathrm{F}$ stars in the complete part of the Hipparcos catalog. Creze et al. (1998) report a local density of matter based on the kinematics of these stars of $0.076 \pm 0.015 M_{\odot} \mathrm{pc}^{-3}$, compared to $0.085 \pm 0.02$ $M_{\odot} \mathrm{pc}^{-3}$ of locally visible material. Using a similiar sample, Holmberg and Flynn (1999) derive $0.102 \pm 0.006 M_{\odot} \mathrm{pc}^{-3}$ in gravitating mass versus $0.093 \pm$ $0.01 M_{\odot} \mathrm{pc}^{-3}$ in visible material. The difference between the estimates of the amount of visible material reflects the observational uncertainty in determining 
this quantity, which is shown (for the Holmberg and Flynn analysis) divided into various components in table 1 . The columns show the component description, the local mass density $\rho(0)$ in $M_{\odot} \mathrm{pc}^{-3}$, the vertical velocity dispersion, $\sigma_{w}$ and the surface density, $\Sigma$ in $M_{\odot} \mathrm{pc}^{-2}$. Broadly, half of the local mass density is in gas and half is in stars and stellar remnants. The most uncertain part of the local density is due to the gas, which is rather poorly constrained because one observes directly the column density of gas along a line of sight, rather than the volume density. The total contribution of the gas to the surface density of matter at the Sun is approximately 30 per cent, because the gas layer is so much thinner than the combined stellar layers.

Table 1. Massive components of the local disk

\begin{tabular}{rllcc}
\hline$i$ & Description & $\begin{array}{l}\rho_{i}(0) \\
M_{\odot} \mathrm{pc}^{-3}\end{array}$ & $\begin{array}{c}\sigma_{i} \\
\mathrm{~km} \mathrm{~s}^{-1}\end{array}$ & $\begin{array}{c}\Sigma_{\odot} \mathrm{Mc}_{\odot} \\
\mathrm{pc}^{-2}\end{array}$ \\
\hline 1 & $\mathrm{H}_{2}$ & 0.021 & 4.0 & 3.0 \\
2 & $\mathrm{HI}(1)$ & 0.016 & 7.0 & 4.0 \\
3 & $\mathrm{H} \mathrm{I}(2)$ & 0.012 & 9.0 & 4.0 \\
4 & warm gas & 0.001 & 40.0 & 2.0 \\
5 & giants & 0.0006 & 17.0 & 0.4 \\
6 & $M_{V}<2.5$ & 0.0031 & 7.5 & 0.9 \\
7 & $2.5<M_{V}<3.0$ & 0.0015 & 10.5 & 0.6 \\
8 & $3.0<M_{V}<4.0$ & 0.0020 & 14.0 & 1.1 \\
9 & $4.0<M_{V}<5.0$ & 0.0024 & 19.5 & 2.0 \\
10 & $5.0<M_{V}<8.0$ & 0.0074 & 20.0 & 6.5 \\
11 & $M_{V}>8.0$ & 0.014 & 20.0 & 12.3 \\
12 & white dwarfs & 0.005 & 20.0 & 4.4 \\
13 & brown dwarfs & 0.008 & 20.0 & 6.2 \\
14 & stellar halo & 0.0001 & 100.0 & 0.6 \\
\hline
\end{tabular}

Sommer-Larsen and Dolgov (1999) have recently used this estimate of the local disk mass to estimate the total baryonic (visible) mass of the Milky Way. This turns out to be only weakly dependent on the adopted scale length of matter in the disk, and for reasonable values of 3 to $5 \mathrm{kpc}$, they derive a mass of $\approx 5 \times 10^{10} M_{\odot}$.

\subsection{New limits on faint stars as disk dark matter}

Prior to the Space Telescope, the contribution of faint stars to the total disk mass was rather poorly constrained (viz. Row 11 of table 1), since the stars are so faint that detecting them from the ground amongst far more numerous galaxies was an arduous task. Space Telescope, with its 0.08 arcsecond spatial resolution, allows routine separation of stars and galaxies to be carried out to as faint as $I=26.3$ (in the Hubble Deep Field; Flynn, Gould and Bahcall 1996). As a result of this and other important advances in ground based techniques (Reid et al. 1995), the faint end of the mass and luminosity function has been probed and constrained much better in the last 5 years. In particular, the faint $\left(M_{V}>8.0\right) \mathrm{M}$ dwarfs are now known to contribute only a minor fraction (circa 25 percent, see Table 1, row 11) to the total disk mass, whereas they could 
potentially have dominated the disk and provided the unseen matter (see e.g. Flynn, Gould and Bahcall 1999).

\section{Baryonic dark matter in the Milky Way halo}

The rotation curve of the Milky Way (and external spiral galaxies) indicates that it may be embedded in a massive, unseen "dark halo", which dominates its mass beyond the solar circle. Standard models of the dark halo contribute about $0.01 M_{\odot} \mathrm{pc}^{-3}$ locally, compared to $0.1 M_{\odot} \mathrm{pc}^{-3}$ for the disk, and have a density fall-off like $R^{-2}$, where $R$ is the Galactocentric radius. The total mass is very uncertain, since it rises with increasing distance from the Galactic center without evidence for a cutoff, but appears to be as much as $10^{12} M_{\odot}$ within $R<200$ kpc (Zaritsky, 1999).

Now that the mass density of the local disk from its vertical kinematics has been well determined at $0.10 \pm 0.01 M_{\odot} \mathrm{pc}^{-3}$, mass modelling indicates that both the dark halo and the disk contribute approximately equally at the solar circle to the local rotation velocity of 180 to $220 \mathrm{~km} \mathrm{~s}^{-1}$ (Fich and Tremaine 1991, Dehnen and Binney, 1998).

A great range of candidates exist which could constitute the dark halo, falling broadly into two classes (at least from an observational astronomer's viewpoint), namely baryonic and non-baryonic candidates, or MACHOs and WIMPs. Machos (Massive Astrophysical Compact Halo Objects) would be baryonic objects which collapsed early in the universe, collecting in massive halos within which a smaller fraction of additional baryonic material cooled, collected and formed the visible components of galaxies (Carr 1994). MACHOs as dark matter candidates has turned from a theoretical to a very active observational field in the last decade since Paczynski's (1986) suggestion that such objects could be detected by microlensing of background sources. Wimps (Weakly Interacting Massive Particles) is a generic term for as yet undetected fundamental particles (non-baryonic) which could make up the dark halo of the Milky Way and also dominate the mass of the Universe on much larger scales as well (Sadoulet, 1999).

\subsection{MACHOs}

Concentrating now on baryonic dark matter candidates, several groups ${ }^{1}$ have large programs running to measure the rate of microlensing toward the Magellanic clouds. Currently, the small number of detected microlensing events imply that about $50 \%$ of the dark halo could be in MACHOs, with best fit masses for the observed events for the dark objects of approximately $0.5 M_{\odot}$ objects (Alcock et al. 1996). Very importantly, light objects $10^{-7}<M / M_{\odot}<10^{-3}$ (e.g. brown dwarfs, Jupiters, planets) are currently ruled out by consideration of the typical duration and the event rate of the microlensing (Alcock et al. 1998).

\footnotetext{
${ }^{1}$ EROS : www.lal.in2p3.fr/recherche/eros/erosa.html MACHO : wwwmacho.anu.edu.au MOA : www.phys.vuw.ac.nz/dept/projects/moa

OGLE : www.astro.princeton.edu/ stanek/ogle
} 
Constraints on what these objects could be have recently been derived using the deepest images taken with the Hubble Space Telescope, the Hubble Deep Field (Flynn, Gould and Bahcall 1999). Star/Galaxy separation is possible to $I=26.3$ in this field. Three groups (Flynn, Gould and Bahcall 1996, Elson, Santiago and Gilmore 1996 and Mendez et al. 1996) found the same 18 stars down to this limit in HDF.

\subsection{Are the MACHOs red dwarfs?}

Flynn, Gould and Bahcall analysed the colour-magnitude diagram of the HDF stars, and found no stars redder than $V-I=1.8$ within 1.6 magnitudes of the $I$-band magnitude limit. If the dark halo of the Galaxy were composed of normal $\mathrm{M}$ dwarfs $(V-I \gtrsim 2)$, such as those found locally in the disk, tens or even hundreds of them would be seen directy in the HDF. Since none were found, this a strict limit of less than 1 percent of the dark halo could be in this form. Even stricter limits (i.e. much less than $1 \%$ ) if the $\mathrm{M}$ dwarfs are assumed to have I band luminosities like those of theoretical models of metal weak (the dark matter presumbably formed from material of near primordial abundance) M dwarfs (Graff and Freese 1996). Red dwarfs appear well ruled out.

\subsection{Are the MACHOs white dwarfs?}

Another candidate for the dark matter is old white dwarfs (Richer 1999). There are a number of problems with these however, because the white dwarf precursors have to be created in a rather specific mass range, i.e. no high mass or low mass stars are born at the same time. Too many high mass stars would pollute the proto-Milky Way gas with heavy elements when they become supernovae, leading to much more heavy elements in the present day Milky Way than is observed, while low mass stars remain for much longer than a Hubble time on the main sequence, and are ruled out directly from counts of red stars in the HDF.

Recently calculated cooling curves for old white dwarfs of primordial abundance indicate that far from getting redder as they age and cool, they become very blue, $0.0<V-I<1.0$ (Hansen 1999). In this case, predictions of the absolute magnitude from models indicate that a few old, blue white dwarfs may very well be present in the Hubble Deep Field at very faint levels (HDF reaches $V \approx 29$ ). Just such objects have been reported very recently after second epoch images of the HDF were taken, revealing themselves as a handful of very faint $(I \approx 28)$, blue $(0<V-I<1)$, moving objects (Ibata et al. 1999). If these objects are old white dwarfs, then they have approximately the right magnitudes, colours, proper motions and number density to account for the matter in the dark halo! A potential difficulty for this solution to the dark matter problem is that significant numbers of such objects might be expected in surveys of high proper motions stars, but are apparently absent (Flynn et al. 2000). Further observations with HST are planned and should clarify the nature of these objects in the near future.

Acknowledgments. This research was supported in part by the Academy of Finland. 


\section{References}

Alcock et al. 1996, ApJ, 471, 774

Alcock et al. 1998, ApJL, 499, L9

Carr, B. 1994, ARA\&A, 32, 531

Creze, M., Chereul, E., Bienayme, O. \& Pichon, C. 1998, A\&A, 329, 920

Einasto \& Einasto, 2000, this volume

Fich, M. \& Tremaine, S. 1991, ARA\&A, 29, 409

Flynn, C., Gould, A. \& Bahcall, J. N. 1996, ApJ, 466, L55

Flynn, C., Gould, A. \& Bahcall, J. 1999, ASP Conf. Ser. 136: Galactic Halos, 387

Flynn, C., Sommer-Larsen, J., Fuchs, B., Graff, D., \& Salim, S. 2000, astro$\mathrm{ph} / 9912264$

Graff, D. S. \& Freese, K. 1996, ApJ, 467, L65

Ibata, R. A., Richer, H., Gilliland, R., Scott, D. 1999, ApJL, 524 L1

Reid, I. N., Hawley, S. L., \& Gizis, J. E. 1995, AJ, 110, 1838

Richer, H. 1999, astro-ph/9906424

Sadoulet, B. 1999, Rev. Modern Phys., Spec. Issue, 71, 197-204 (1999), 71, 197

Sommer-Larsen, J. and Dolgov, A, 1999, astro-ph/9912166

Van Den Bergh, S. 1999, PASP, 111, 657

Zaritsky, D. 1999, ASP Conf. Ser. 136: Galactic Halos, 34 